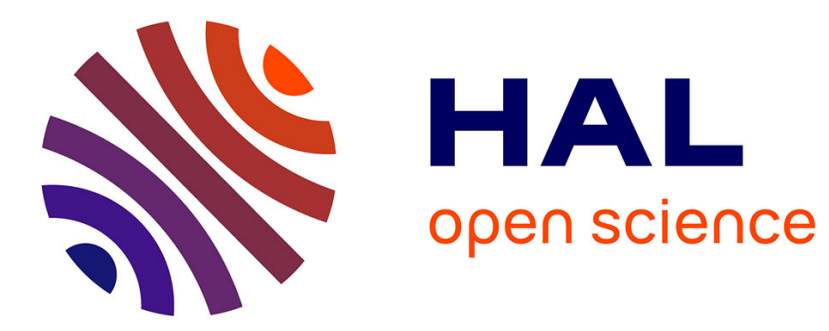

\title{
6-axis inertial sensor using cold-atom interferometry
}

B. Canuel, F. Leduc, D. Holleville, Alexandre Gauguet, J. Fils, A. Virdis, A. Clairon, N. Dimarcq, Ch. J. Borde, Philippe Bouyer, et al.

\section{To cite this version:}

B. Canuel, F. Leduc, D. Holleville, Alexandre Gauguet, J. Fils, et al.. 6-axis inertial sensor using coldatom interferometry. Physical Review Letters, 2006, 97, pp.010402. 10.1103/PhysRevLett.97.010402 . hal-00106373

\section{HAL Id: hal-00106373 \\ https://hal.science/hal-00106373}

Submitted on 11 Apr 2016

HAL is a multi-disciplinary open access archive for the deposit and dissemination of scientific research documents, whether they are published or not. The documents may come from teaching and research institutions in France or abroad, or from public or private research centers.
L'archive ouverte pluridisciplinaire HAL, est destinée au dépôt et à la diffusion de documents scientifiques de niveau recherche, publiés ou non, émanant des établissements d'enseignement et de recherche français ou étrangers, des laboratoires publics ou privés. 


\title{
Six-Axis Inertial Sensor Using Cold-Atom Interferometry
}

\author{
B. Canuel, F. Leduc, D. Holleville, A. Gauguet, J. Fils, A. Virdis, * A. Clairon, N. Dimarcq, Ch. J. Bordé, and A. Landragin ${ }^{\dagger}$ \\ LNE-SYRTE, CNRS UMR 8630, Observatoire de Paris, 61 avenue de l'Observatoire, 75014 Paris, France \\ P. Bouyer \\ Laboratoire Charles Fabry, CNRS UMR 8501, Centre Scientifique d'Orsay, Bâtiment 503, Bô̂te Postale 147, 91403 Orsay, France
} (Received 14 March 2006; published 7 July 2006)

\begin{abstract}
We have developed an atom interferometer providing a full inertial base. This device uses two counterpropagating cold-atom clouds that are launched in strongly curved parabolic trajectories. Three single Raman beam pairs, pulsed in time, are successively applied in three orthogonal directions leading to the measurement of the three axis of rotation and acceleration. In this purpose, we introduce a new atom gyroscope using a butterfly geometry. We discuss the present sensitivity and the possible improvements.

PACS numbers: 03.75.Dg, 06.30.Gv, 39.20.+q
\end{abstract}

Since its proof of principle in 1991 [1,2], atom interferometry has demonstrated, in particular, great sensitivity to accelerations [3,4] and rotations [5,6]. Among beautiful applications [7], these experiments offer attractive perspectives for application in inertial navigation, geophysics, or tests of fundamental physics [8], where the ability of coldatom interferometry to give stable and accurate measurements can bring a real improvement compared to standard technologies, as is already the case for atomic clocks [9]. Nowadays, best performances are achieved by interferometers using optical transitions [10,11], based on a sequence of three Raman pulses $(\pi / 2-\pi-\pi / 2)$ first introduced by Kasevich and Chu [2]. The pulses couple the two hyperfine ground states $\left(\mid 6 S_{1 / 2}, F=3, m_{F}=0>\right.$ and $\mid 6 S_{1 / 2}, F=4, m_{F}=0>$ in the case of Cesium atoms), which split apart when using counterpropagating Raman lasers [12]. The $\pi / 2$ and $\pi$ pulses realize, respectively, the beam splitters and mirrors of the interferometer. This configuration allows measurement of acceleration along the direction of propagation of the Raman lasers. When the geometrical area included in the interferometer is nonzero, it also gives access to the rotation around the axes perpendicular to the oriented area. Up to now, atom interferometers have only been proven to be sensitive to a single inertial quantity (e.g., acceleration or rotation along one single axis), although intrinsically sensitive to at least both acceleration and rotation. In order to get full inertial monitoring, all six axes ( 3 rotations and 3 accelerations) must be measured, as needed for inertial navigation, geophysics measurements, or some tests of fundamental physics [8]. In the past, this was achieved by implementing multiple inertial sensors, as proposed in [8], and the ability of using a single "proof mass" for measuring all inertial axis has not yet been achieved. This represents a real challenge for inertial measurement such as the possibility of monitoring gravity and the 3 components of the earth rotation at the same position.

In this Letter we describe a new setup which is sensitive along six axes of inertia. The two key features of our setup are the use of a single Raman beam pair pulsed in time and the choice of a strongly curved parabolic trajectory. This allows successive use of three configurations of Raman lasers that interact with two counterpropagating atomic clouds, giving access to all components of rotation and acceleration. For one of these components, we use a new butterfly configuration based on a four-pulse sequence $(\pi / 2-\pi-\pi-\pi / 2)$. In addition, we introduce an original Raman configuration to reduce the systematic effect introduced by wave front distortions.

In our experiment, about $10^{7}$ Cesium atoms are trapped from a vapor in magneto-optical traps during $125 \mathrm{~ms}$, and cooled down to $3 \mu \mathrm{K}$. The Cesium clouds are launched along parabolic trajectories using moving molasses at $2.4 \mathrm{~m} \cdot \mathrm{s}^{-1}$, with an angle of $8^{\circ}$ with respect to the vertical direction. Then the atoms are prepared in the state $\mid 6 S_{1 / 2}$, $F=3, m_{F}=0>$ before entering the interferometer zone at the top of their trajectory, where they interact with the Raman lasers. In the following, $\mathbf{k}$ denotes the effective wave vector of the Raman transition, and $\varphi_{l}$ the difference of phase between the two lasers. The interrogation sequence is achieved with a single pair of Raman beams covering the entire interrogation zone. The beams are switched on during $20 \mu$ s to realize the Raman pulses, which provides an easy way to change the pulse sequence. The atomic velocity and the Raman beam size, $30 \mathrm{~mm}$ diameter $\left(1 / e^{2}\right)$, set the maximum interrogation time to $80 \mathrm{~ms}$. At the exit of the interferometer, the transition probability depends on the inertial forces through the phase difference accumulated between the two arms of the interferometer [13]. Raman transitions enable detection of the internal states of the atoms by fluorescence imaging.

We now present the description of the 6 axis inertial sensor principle. The direction of sensitivity of the setup is defined by the direction of the Raman interrogation laser with respect to the atomic trajectory. As illustrated in Fig. 1, with a classical three pulses sequence $(\pi / 2-\pi-$ $\pi / 2$ ), a sensitivity to vertical rotation $\Omega_{z}$ and to horizontal acceleration $a_{y}$ is achieved by placing the Raman lasers 


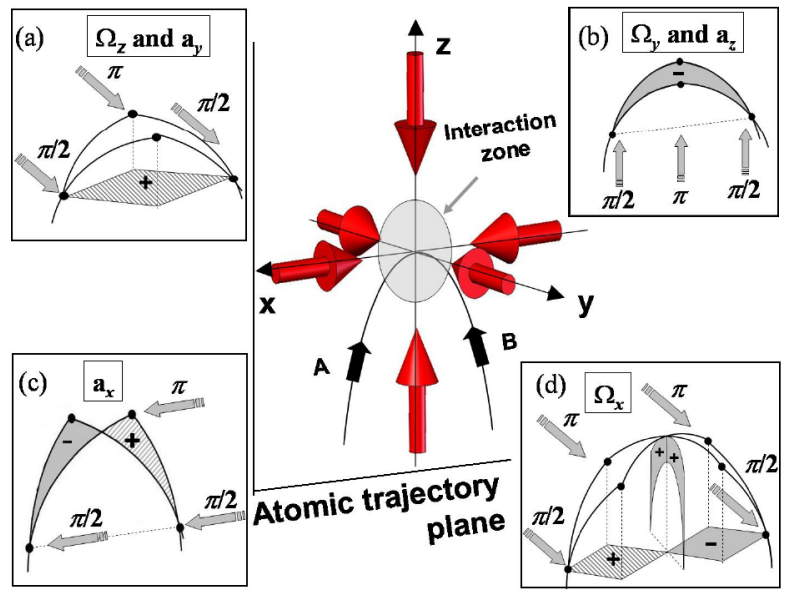

FIG. 1 (color online). Six-axis inertial sensor principle. The atomic clouds are launched on a parabolic trajectory, and interact with the Raman lasers at the top. The four configurations (a)(d) give access to the 3 rotations and the 3 accelerations. In the three pulses configuration, the Raman beams direction can be horizontal or vertical, creating the interferometer in a horizontal (a) or vertical (b),(c) plane. With a butterfly four-pulse sequence of horizontal beams (d), the rotation $\Omega_{x}$ can be measured.

horizontal and perpendicular to the atomic trajectory [5] [Fig. 1(a)]. The same sequence, using vertical lasers, leads to the measurement of horizontal rotation $\Omega_{y}$ and vertical acceleration $a_{z}$ [Fig. 1(b)]. Thanks to our specific setup, we also have access to the other components of acceleration and rotation which lie along the horizontal direction of propagation of the atoms ( $x$ axis). The use of cold atoms in strongly curved trajectories allows us to point the Raman lasers along the $x$ direction, offering a sensitivity to acceleration $a_{x}$ and no sensitivity to rotation [Fig. 1(c)]. We also have an easy access to the horizontal rotation $\Omega_{x}$ by changing the pulse sequence to 4 pulses: $\pi / 2-\pi-\pi-$ $\pi / 2$ [Fig. 1(d)]. We detail in the following the two configurations: the classical three pulses sequence (a) and our new butterfly four-pulse sequence (d).

The first pulse sequence that we study here is a standard three pulses $(\pi / 2-\pi-\pi / 2)$. The phase shift depends on the acceleration $\mathbf{a}$ and on the rotation rate $\boldsymbol{\Omega}$ through [11]:

$$
\Delta \Phi=\mathbf{k}[\mathbf{a}-2(\mathbf{\Omega} \times \mathbf{v})] T^{2} .
$$

The scale factor depends only on $\mathbf{k}, 2 T$ the total interrogation time and $\mathbf{v}$ the mean velocity in the laboratory frame, which are well controlled. In the following, $\mathbf{k}$ is horizontal and along the $y$ axis, as we see in Fig. 1(a). The surface delimited by the two arms of the interferometer is curved and the projection of the oriented area on the two vertical planes cancels out. Therefore it gives access to accelerations along this direction and to rotations around the vertical axis. To discriminate between acceleration and rotation, we use two counterpropagating cesium atomic clouds leading to an opposite velocity in Eq. (1) [14].
In our setup, we have developed a new method to reduce the variations of the local wave vector $\mathbf{k}$, which induce perturbations that can be read as inertial phase shifts [15]. In this method the Raman beams propagate in the same optical system with orthogonal circular polarizations, pass through the atomic trajectories, and are retroreflected through a quarter-wave plate [16]. In this case, the aberrations are common and compensated most of the time: until the lasers cross the atoms. With circular polarizations, the atoms can experience two diffraction processes with opposite $\mathbf{k}$ vectors. In order to select a single diffraction process, we tilt the laser beams by $6^{\circ}$ in the horizontal plane (Fig. 2), and compensate the Doppler effect by an additional frequency difference between the Raman lasers [17]. Since the two atom clouds are counterpropagating, their Doppler detunings are opposite, which means that each atomic cloud is resonant with a different Raman pair, and this results in an opposite effective wave vector for the two interferometers. Therefore, the rotation and the acceleration parts are, respectively, obtained by the sum and the difference of the phases measured by the two interferometers (A and $\mathrm{B})$.

We show in Fig. 3 the scan of the fringes of both interferometers by changing the phase $\varphi_{l}$ between the first and the second Raman pulse. With our interrogation time of $2 T=60 \mathrm{~ms}$, the fringe contrasts are, respectively, $14.4 \%$ and $10.6 \%$ for A and B. The low contrast values can be explained by the sizes of the clouds after ballistic expansion ( $3.3 \mathrm{~mm}$ rms radius) and by the Gaussian intensity profile of the laser beams. In addition, mismatch between the trajectories $\mathrm{A}$ and $\mathrm{B}$ requires a compromise for the diffraction efficiency that leads to a reduction of the contrast by a factor of about 2 .

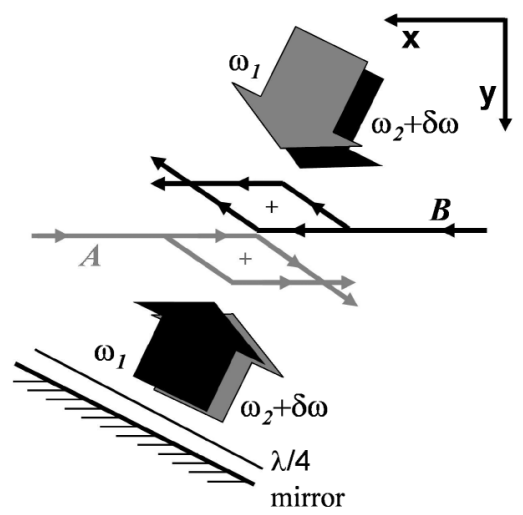

FIG. 2. The orthogonally polarized copropagating Raman beams are tilted with respect to the atom trajectories. They are retroreflected by a mirror through a quarter-wave plate so that the atoms interact with counterpropagating beams at frequency $\omega_{1}$ and $\omega_{2}+\delta \omega$ with $\omega_{1}-\omega_{2} \approx 9.2 \mathrm{GHz}$. The detuning $\delta \omega$ compensates for the Doppler shift so that each of the two counterpropagating atom clouds can interact with only one pair of beams. Interferometer areas are shown in the case of a three-pulse interferometer. 


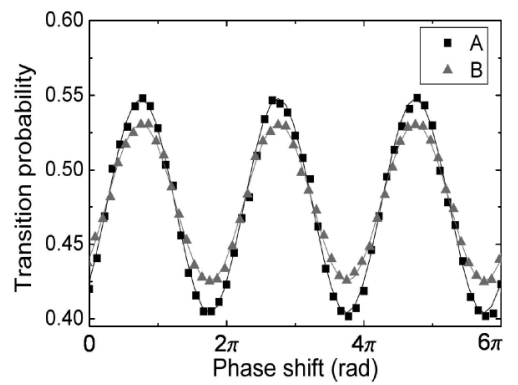

FIG. 3. Fringes obtained with the two interferometers A and B, for an interrogation time of $2 T=60 \mathrm{~ms}$ and a cycling time of $560 \mathrm{~ms}$ without averaging.

To reach the maximum sensitivity to inertial forces, we operate the interferometer on the side of a fringe. To realize this condition for the two interferometers together, we align the Raman laser in the horizontal plane and compensate the rotational phase with an appropriate change of $\varphi_{l}$. In addition, by using two different values of $\varphi_{l}$, the interferometers can sit alternately on each side of a fringe [9], which allows rejection of long-term drifts of the contrast and of the offset of the fringe patterns. Figure 4 shows the time recordings of vertical rotation $\Omega_{z}$ and horizontal acceleration $a_{y}$ extracted from the half sum and half difference of the two interferometers' phase shifts.

These results were obtained using an isolation platform (nano-K 350BM-1) to reduce the level of vibration in order to reach the maximum sensitivity [18]. However, this system introduces long-term tilt fluctuations which yield some acceleration fluctuations through the projection of $\mathbf{g}$ on the direction of $\mathbf{k}$. To limit this effect, we have developed a servo-lock of the platform tilt. The residual oscillation of this system at $0.03 \mathrm{~Hz}$ can be identified on the acceleration signal. Since this oscillation completely disappears on the rotation signal, it gives a clear validation of

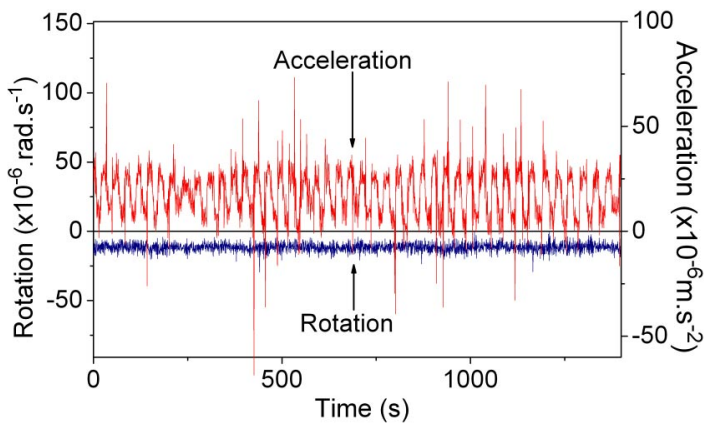

FIG. 4 (color online). Acceleration and rotation signals extracted from the half sum and half difference of the phase shifts of interferometers $\mathrm{A}$ and $\mathrm{B}$. To obtain the actual value of the rotation, the phase $\varphi_{l}$ has to be taken into account. dc offset on the acceleration signal is due to the residual contribution of gravitational acceleration. The acceleration dispersion on the acceleration signal comes from oscillations of the isolation platform. the discrimination concept. We estimate the performances of our setup from the Allan standard deviation of these measurements. The signal-to-noise ratio from shot to shot $(0.56 \mathrm{~s})$ is 12 for the acceleration and 39 for the rotation leading to a respective sensitivity of $4.7 \times 10^{-6} \mathrm{~m} \cdot \mathrm{s}^{-2}$ and $2.2 \times 10^{-6} \mathrm{rad} \cdot \mathrm{s}^{-1}$ for $1 \mathrm{~s}$ averaging time. For both measurements, the Allan standard deviation (Fig. 5) approaches the typical white noise behavior for long integration times. The sensitivity reaches $6.4 \times 10^{-7} \mathrm{~m} \cdot \mathrm{s}^{-1}$ for acceleration and $1.4 \times 10^{-7} \mathrm{rad} \cdot \mathrm{s}^{-1}$ for rotation after $10 \mathrm{~min}$ of averaging time.

We have performed the measurement of the Earth's rotation rate with our cold-atom interferometer: $5.50 \pm$ $0.05 \times 10^{-5} \mathrm{rad} \cdot \mathrm{s}^{-1}$, in which the error bar corresponds to statistical uncertainty. This measured value for the projection along the vertical axis was found in good agreement with the expected value at Paris latitude $\left(\lambda=48^{\circ} 50^{\prime} 08^{\prime \prime}\right)$ : $5.49 \times 10^{-5} \mathrm{rad} \cdot \mathrm{s}^{-1}$.

We now turn to the butterfly configuration [Fig. 1(d)], which was first proposed to measure the gravity gradient [19]. It can be used to measure rotations with the same Raman beams as in the previous configuration ( $y$ axis) but in a direction ( $x$ axis) that cannot be achieved with a standard 3 pulses sequence. Four pulses, $\pi / 2-\pi-\pi-$ $\pi / 2$, are used, separated by times $T / 2-T-T / 2$, respectively. The atomic paths cross each other leading to a twisted interferometer. The horizontal projection of the oriented area cancels out so that the interferometer is insensitive to rotation around the $z$ axis. In contrast, the vertical projection now leads to a sensitivity to rotation around the $x$ axis:

$$
\Delta \Phi=\frac{1}{2}[\mathbf{k} \times(\mathbf{g}+\mathbf{a})] \Omega \mathrm{T}^{3} .
$$

This sensitivity to rotation appears from a crossed term with acceleration and is no longer dependent on the launching velocity. This configuration is not sensitive to dc accelerations along the direction of the Raman laser, but remains sensitive to fluctuations of horizontal and vertical acceleration. With our isolation platform, the remaining fluctuations are negligible compared to $\mathbf{g}$, which does not

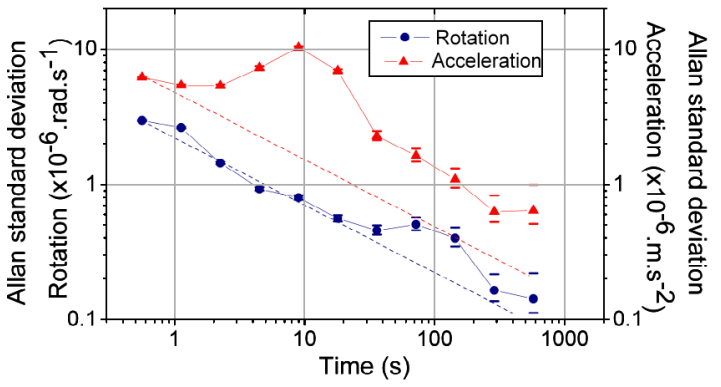

FIG. 5 (color online). Allan standard deviations of acceleration (triangles) and rotation (circles) measurements. Dashed lines corresponds to the $-1 / 2$ slope expected for a white noise. The peak near $10 \mathrm{~s}$ averaging time on the acceleration signal is due to the residual oscillations of the isolation platform tilt. 


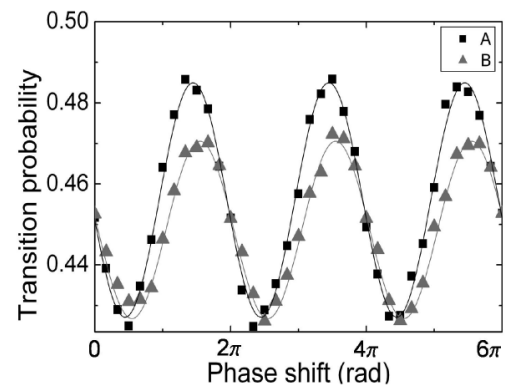

FIG. 6. Fringes obtained with both interferometers A and B in the four-pulses butterfly configuration for a total interrogation time of $2 T=60 \mathrm{~ms}$.

compromise the stability of the scaling factor. The sensitivity to rotation is comparable with that of configurations (a) and (b). With $2 T=60 \mathrm{~ms}$, this configuration leads to a interferometer area reduced by a factor 4.5 , but it scales with $T^{2}$ and thus would present a higher sensitivity for longer interrogation times.

The atomic fringe patterns are presented in Fig. 6 and show contrasts of $4.9 \%$ and $4.2 \%$ for interferometer $A$ and $\mathrm{B}$, respectively. By operating the interferometer on the fringe side, as explained before, we obtain a signal-to-noise ratio from shot to shot of 18 limited by the residual vibrations. The sensitivity to rotation is equal to $2.2 \times$ $10^{-5} \mathrm{rad} \cdot \mathrm{s}^{-1}$ in $1 \mathrm{~s}$, decreasing to $1.8 \times 10^{-6} \mathrm{rad} \cdot \mathrm{s}^{-1}$ after $280 \mathrm{~s}$ of averaging time.

To summarize, we have presented the ability to measure the 6 inertial axis with the same setup. This shows the advantage of using cold atoms combined with a single laser beam pulse in the time domain. A first measurement, in three pulses interferometer, has demonstrated a sensitivity of $1.4 \times 10^{-7} \mathrm{rad} \cdot \mathrm{s}^{-1}$ to rotation and $6.4 \times 10^{-7} \mathrm{~m} \cdot \mathrm{s}^{-2}$ to acceleration in $10 \mathrm{~min}$ averaging time. We have measured the Earth's rotation rate with an accuracy of $1 \%$. Many improvements, such as the cold-atom sources, will allow us to increase the sensitivity by a factor of 50 on rotation and 10 on acceleration [20].

We plan to improve the cooling system in order to increase the total number of atoms, to get a lower temperature $(\approx 1 \mu \mathrm{K})$, and to obtain a good superposition of the atomic trajectories.

In addition, we have demonstrated the butterfly configuration, which uses four pulses and which is sensitive to rotation around the axis parallel to the direction of propagation of the atoms at the top of their trajectory. This configuration is especially well adapted to trajectories close to those of an atomic fountain, in which a single source of atom is launched vertically. Since the interferometer area scales with $T^{3}$, this opens the possibility of a cold-atom gyroscope reaching a sensitivity of $10^{-9} \mathrm{rad}$. $\mathrm{s}^{-1}$ in $1 \mathrm{~s}$.

The authors would like to thank the Déléguation Générale pour l'Armement, the Centre National d'Etudes
Spaciales, the SAGEM, the European Union (FINAQS), and the Ile de France region (IFRAF) for their financial supports, Pierre Petit and Christophe Salomon for their contributions to the early stage of the experiment, and Robert Nyman for careful reading of the manuscript.

*Present address: SAGEM Défense et sécurité, Groupe SAFRAN, URD11, 72-74 rue de la Tour Billy, Boîte Postale 72-95101 Argenteuil Cedex, France.

Corresponding author. Electronic address: arnaud.landragin@obspm.fr

[1] O. Carnal and J. Mlynek, Phys. Rev. Lett. 66, 2689 (1991); D. W. Keith, C. R. Ekstrom, Q. A. Turchette, and D.E. Pritchard, Phys. Rev. Lett. 66, 2693 (1991); F. Riehle, Th. Kister, A. Witte, J. Helmcke, and Ch. Bordé, Phys. Rev. Lett. 67, 177 (1991); F. Shimizu, K. Shimizu, and H. Takuma, Phys. Rev. A 46, R17 (1992).

[2] M. Kasevich and S. Chu, Phys. Rev. Lett. 67, 181 (1991).

[3] A. Peters, K. Y. Chung, and S. Chu, Metrologia 38, 25 (2001).

[4] J. M. McGuirk, G. T. Foster, J. B. Fixler, M. J. Snadden, and M. A. Kasevich, Phys. Rev. A 65, 033608 (2002).

[5] T. L. Gustavson, P. Bouyer, and M. A. Kasevich, Phys. Rev. Lett. 78, 2046 (1997).

[6] A. Lenef, T. D. Hammond, E. T. Smith, M. S. Chapman, R. A. Rubenstein, and D. E. Pritchard, Phys. Rev. Lett. 78, 760 (1997).

[7] Atom Interferometry, edited by R. Paul Berman (Academic, London, 1997), and references therein.

[8] R. Bingham et al., Assessment Study Report, ESA-SCI (2000) 10, and references therein.

[9] A. Clairon, Ph. Laurent, G. Santarelli, S. Ghezali, S. N. Lea, and M. Bahoura, IEEE Trans. Instrum. Meas. 44, 128 (1995).

[10] Ch. J. Bordé, Phys. Lett. A 140, 10 (1989).

[11] Ch. J. Bordé, in Laser Spectroscopy X, edited by M. ducloy, E. Giacobino, and G. Camy (World Scientific, Singapore, 1991), Vol. 239.

[12] M. Kasevich, D. S. Weiss, E. Riis, K. Moler, S. Kasapi, and S. Chu, Phys. Rev. Lett. 66, 2297 (1991).

[13] Ch. J. Bordé, Gen. Relativ. Gravit. 36, 475 (2004).

[14] T.L. Gustavson, A. Landragin, and M.A. Kasevich, Classical Quantum Gravity 17, 2385 (2000).

[15] J. Fils, F. Leduc, P. Bouyer, D. Holleville, N. Dimarcq, A. Clairon, and A. Landragin, Eur. Phys. J. D 36, 257 (2005).

[16] A. Landragin and P. Featonby, French Patent No. FR 0215454.

[17] With cold atoms, the Doppler width is small enough so that atoms can only feel the effect of a single Raman pair.

[18] Without the platform, the fluctuations could induce phase shifts higher than $2 \pi$ [3], which would compromise the discrimination process.

[19] T. Gustavson, Ph.D. thesis, Stanford University, 2000.

[20] P. Cheinet, F. Pereira Dos Santos, T. Petelski, J. Le Gouët, K. T. Therkildsen, A. Clairon, and A. Landragin, physics/ 0510261 (to be published). 